\title{
Magnetic resonance imaging is more accurate than mammography for assessing response to neoadjuvant chemotherapy in a breast cancer patient with significant calcifications
}

\author{
Sam C Wang' \\ Bonnie $\mathrm{N}$ Joe 2 \\ Laura J Esserman' \\ 'Carol Franc Buck Breast Care \\ Center, Department of Surgery, \\ University of California, San Francisco, \\ San Francisco, CA, USA; ${ }^{2}$ Department \\ of Radiology, University of California \\ San Francisco, San Francisco, CA, USA
}

Correspondence: Laura J Esserman LJE, Carol Franc Buck Breast Care Center, 2nd Floor, 1600 Divisadero Street, San

Francisco, CA 94II5, USA

$\mathrm{Tel}+|4| 5885769 \mid$

Fax + I $415353957 \mid$

Email laura.esserman@ucsfmedctr.org

\begin{abstract}
For patients with operable breast cancer, neoadjuvant chemotherapy can increase the chance for breast-conserving resection without sacrificing survival length. Breast imaging is an important tool for following disease progression in patients undergoing preoperative therapy. We present a patient who had significant calcification associated with her primary tumor and axillary lymph node disease. After undergoing a course of chemotherapy that was truncated due to side effects, mammogram showed these deposits remained unchanged even though magnetic resonance imaging (MRI) demonstrated that she had a complete response at both sites. Indeed on final pathologic examination there was no invasive disease remaining in the breast and axillary lymph nodes. This case shows the potential superiority of MRI relative to mammograms in assessing the therapeutic response of breast cancer associated with calcifications after neoadjuvant chemotherapy.
\end{abstract}

Keywords: breast cancer, calcifications, neoadjuvant chemotherapy, magnetic resonance imaging

\section{Background}

Greater numbers of breast cancer patients are receiving neoadjuvant chemotherapy (NAC). Large prospective randomized trials comparing preoperative and postoperative therapies in patients with primarily operable disease have demonstrated no differences in disease-free and overall survival between the two groups. ${ }^{1,2}$ These favorable results have enabled providers to offer more patients the chance at breast-conserving operations. Patients with tumors previously thought to be resectable with only full mastectomies now have the option first to undergo NAC in an attempt to shrink the tumor. If that approach proved successful, patients would require only partial mastectomies and better cosmetic results would be achieved without compromising survival. ${ }^{1,2}$

All patients evaluated for NAC require a meticulous staging work up. Physical examination is important for assessing tumor size, location in the breast (as determined by the distance from and position relative to the nipple), and regional lymph node status. Breast imaging, such as by mammography or ultrasound, determines the extent of primary disease and evaluates for synchronous lesions in the ipsilateral and contralateral breasts. Recent studies have shown that magnetic resonance imaging (MRI) may be superior to mammography or ultrasound in discerning the full extent of disease. ${ }^{3,4}$

We describe a case of a breast cancer patient who underwent NAC. Her primary tumor and lymph node disease were associated with extensive calcifications that were unchanged after treatment and remained suspicious for malignancy on post-therapy mammograms. In contrast, post-treatment MRI showed complete tumor response that 
was confirmed on postoperative pathologic examination. This case provides two important lessons. First, calcifications that remain in the breast and lymph nodes after NAC may not necessarily contain disease. Second, breast MRI may be more accurate than mammography for assessing breast cancer response after NAC.

\section{Case presentation}

A 59-year-old woman with a past medical history significant for only hypertension noted a mass in her right breast one month prior to presentation at our clinic. A mammogram ordered by her primary care physician showed suspicious calcifications spanning $5 \mathrm{~cm}$ in the upper outer quadrant of her right breast and also in enlarged axillary lymph nodes (Figure 1). A physical examination performed in our clinic showed the skin around the right nipple was erythematous and thickened and the breast tissue was macronodular and dense. There was an $11 \times 10 \mathrm{~cm}$ mass in the upper outer quadrant of the right breast and a $5 \times 5 \mathrm{~cm}$ area of matted lymph nodes in the axilla. The rest of the examination was otherwise unremarkable.

An ultrasound-guided core biopsy of the right breast mass was obtained and pathologic examination showed invasive ductal carcinoma with micropapillary features. The tumor was negative for estrogen and progesterone receptors, but positive for HER2/neu overexpression. Bilateral breast MRI showed multiple irregular enhancing spiculated masses with linear irregular enhancement in the right outer breast (Figure 2). The largest lesion measured $3.5 \mathrm{~cm}$. Bulky axillary adenopathy was also noted, with the largest lymph node measuring $1.8 \mathrm{~cm}$. There was diffuse skin thickening that appeared to be secondary to venous congestion. The left breast and axilla were unremarkable. Whole body positron emission tomography-computed tomography showed avid uptake of ${ }^{18} \mathrm{~F}$ flurodeoxyglucose in the right breast mass and right axillary lymph nodes. There was no evidence of metabolically active disease in the rest of the body.

Our multidisciplinary care group, which includes surgeons, medical oncologists, and radiation oncologists, recommended that she undergo NAC. However, infusions of doxorubicin and cyclophosphamide had to be stopped after two cycles due to neutropenia and thrombocytopenia. She was switched to paclitaxel and trastuzumab (Herceptin) but developed intolerable mucositis, weight loss, and fatigue by her third cycle. At this point, mammography showed extensive segmental pleomorphic calcifications within the right upper outer quadrant that were unchanged from her pre-treatment mammogram, consistent with known malignancy (Figure 3). Calcifications were also noted in the right axillary lymph nodes that no longer appear enlarged and within thickened periareolar skin. In contrast, MRI showed no residual abnormal enhancement in the breast and resolution of lymphadenopathy (Figure 4).

The patient underwent surgical resection without completing her scheduled chemotherapy cycles. Because she was extremely thin, had pendulous breasts, and the calcifications were located in the right outer quadrant, complete excision of all calcifications would have left a cosmetically unsatisfactory result. We opted for a right wire-bracketed partial mastectomy via a breast reduction technique, right axillary lymph node dissection, and contralateral breast reduction and mastopexy. The procedure on the contralateral breast maintained breast symmetry, whereas reducing the skin envelope on the right breast was thought to help decrease the risk of radiation-induced skin complications and also allowed for the removal for greater than $95 \%$ of the calcifications in a specimen that measured approximately $10 \times 20 \mathrm{~cm}$.

The final pathologic examination showed focal residual ductal carcinoma in situ (DCIS) scattered over $5 \mathrm{~cm}$, but with no invasive component. Microcalcifications were found throughout benign ducts, DCIS, and stroma. Sclerosing adenosis was present. The skin had no pathologic abnormalities. There were 11 nodes in the specimen and none had tumor.

Postoperatively, the patient had an unremarkable recovery and underwent radiation therapy approximately one month after her operation. She continued trastuzumab monotherapy for one year at which point a follow-up MRI was performed and showed no recurrence (data not shown).

\section{Discussion}

Neoadjuvant chemotherapy (NAC) is becoming an increasingly important treatment option for breast cancer patients. It offers more women the chance at breast-conserving resection without compromising survival. ${ }^{1,2}$ Furthermore, tumor response to NAC predicts survival length and thus provides prognostic information. ${ }^{1}$ For our patient, it was a favorable sign that she had such a positive response to a truncated therapy regimen. Even though her tumors showed HER2/neu overexpression, which is associated with poorer prognosis, these lesions are responsive to chemotherapy. Previous studies have shown that combination treatment with trastuzumab in a neoadjuvant setting can increase significant pathologic response from $25 \%-47 \%$ to $67 \%-73 \%$. $^{5,6}$

Traditional techniques to monitor clinical response to NAC include physical examination, ultrasound, and mammography. However, a recent study showed that 

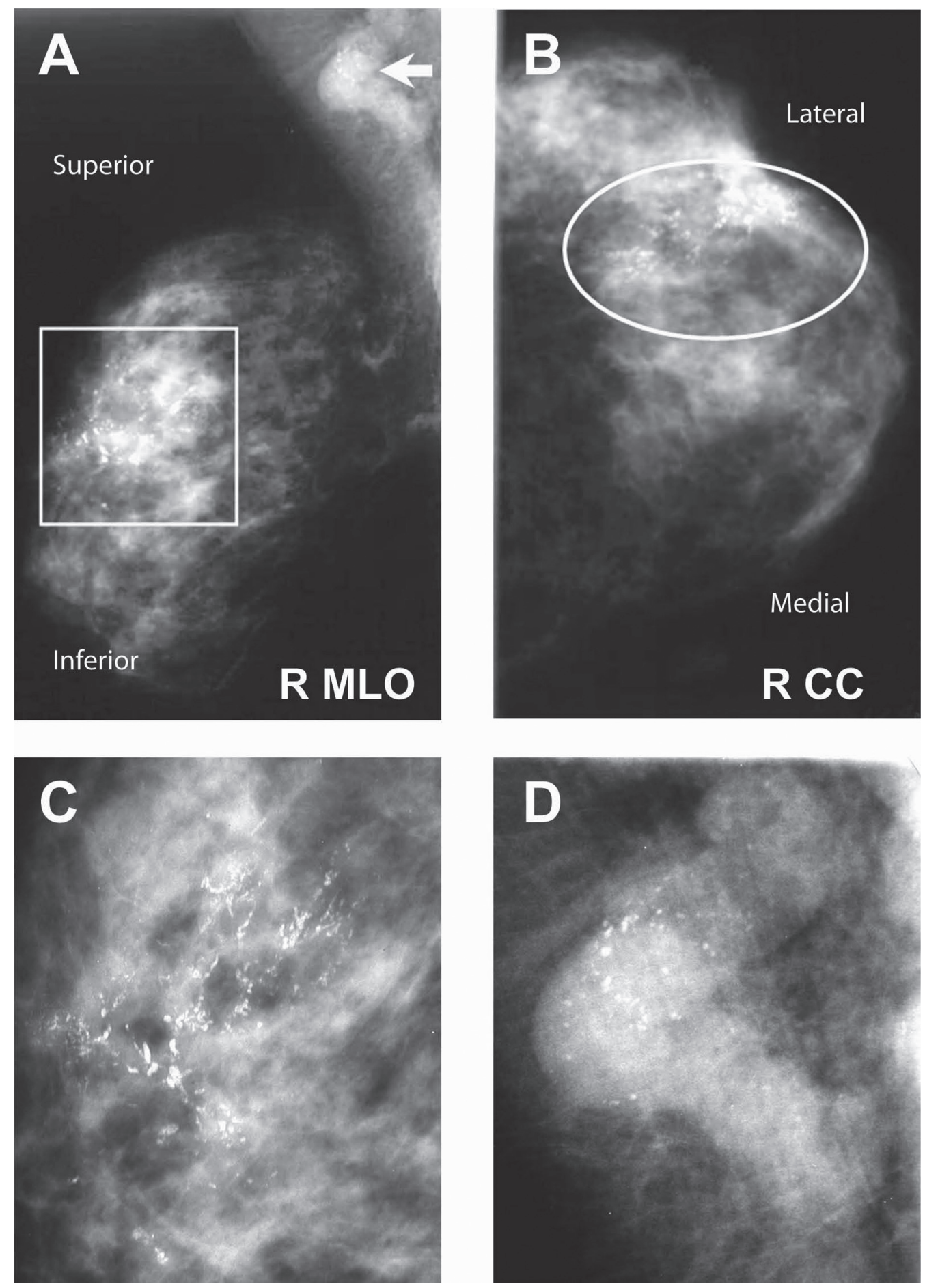

Figure I Right breast mammogram pre-treatment. Suspicious calcifications are seen in the right upper outer breast on A) mediolateral-oblique (MLO; rectangle) and B) craniocaudal (CC; oval) views. Zoomed-in view of area within rectangle in A shows fine linear and branching calcifications in a segmental distribution C). Zoomed-in view of enlarged, calcified axillary lymph node denoted by white arrow in A D).

these techniques are accurate to within one centimeter of actual tumor diameter as determined on final pathology examination in only $66 \%-75 \%$ of patients. ${ }^{7}$ We have previously shown that MRI-measured tumor response to neoadjuvant chemotherapy has a higher correlation to final pathology and may also be able to predict survival length.,8 This finding appears to be substantiated by the currently on-going multilocation, prospective ACRIN 6657/CALGB $150007 / 150012$ study of women undergoing neoadjuvant therapy. Preliminary data shows that MRI volume and MRI 

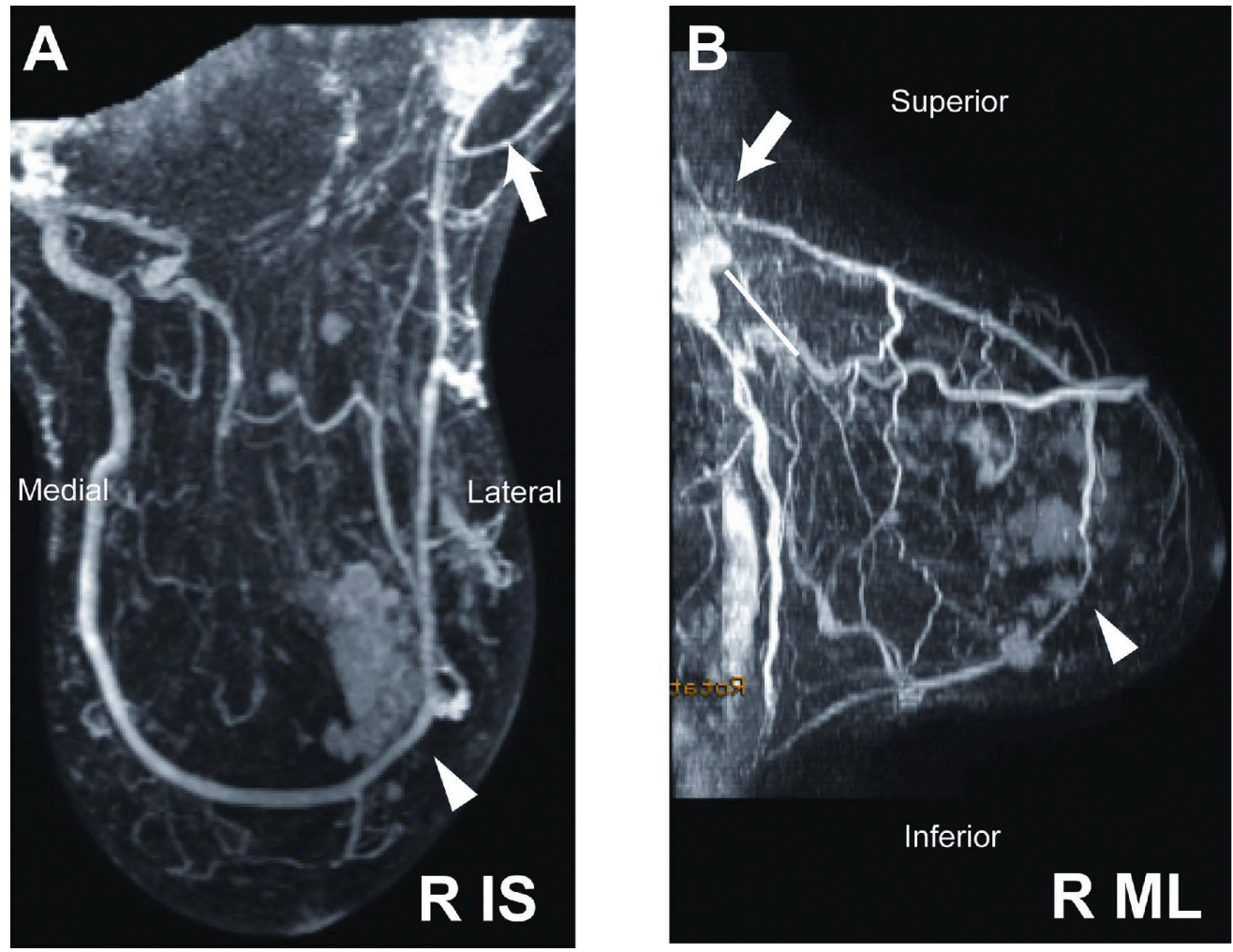

Figure 2 Right breast magnetic resonance imaging pre-treatment. Three-dimensional maximal intensity projection reformat of the right breast images in $\mathbf{A}$ ) inferior-superior and B) mediolateral projections. Multiple irregular spiculated enhancing masses are seen with the dominant lesion measuring up to $3.5 \mathrm{~cm}$, centered within the retroareolar breast (arrowhead). Right axillary lymphadenopathy with largest node measuring $1.8 \mathrm{~cm}$ (arrow).
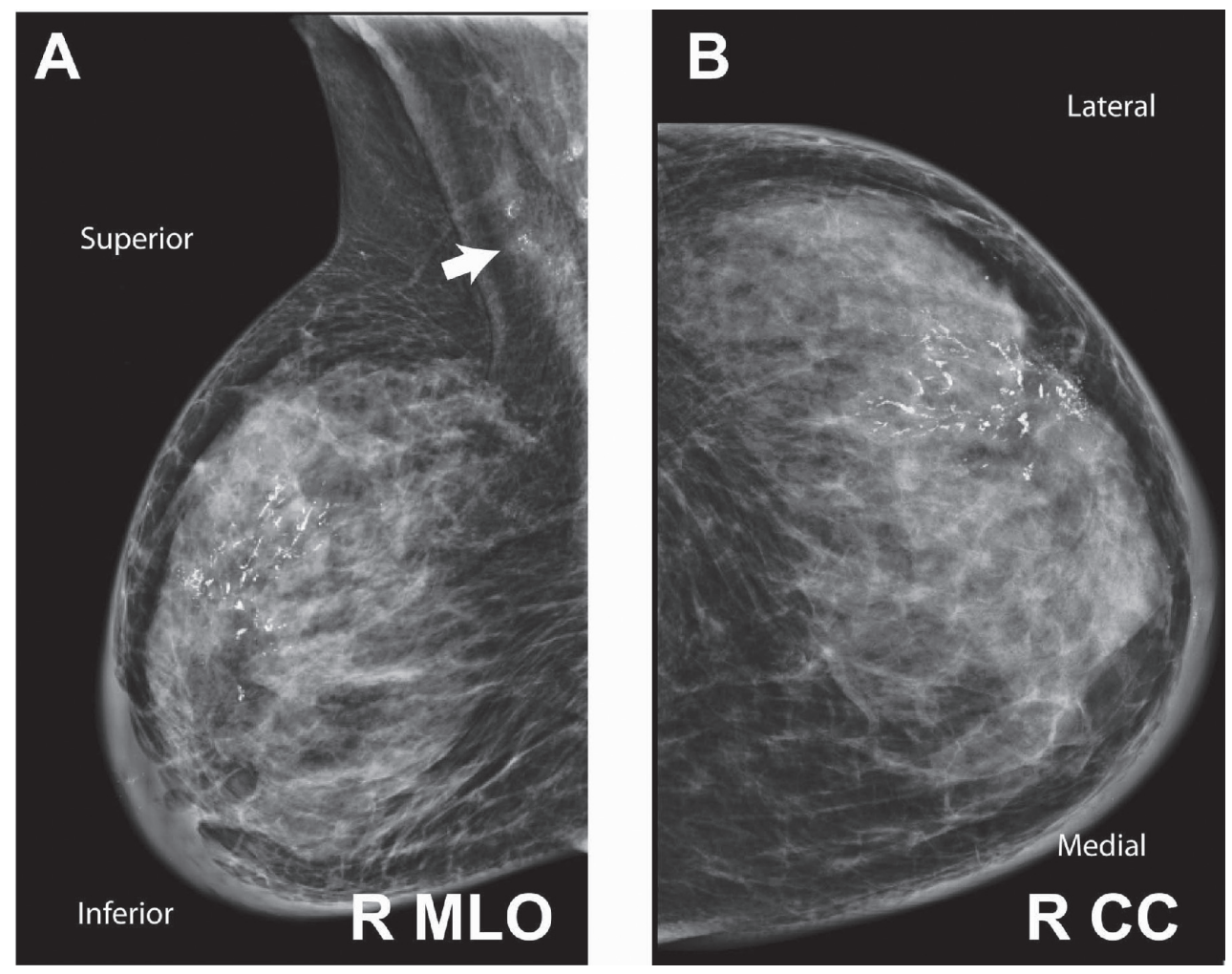

Figure 3 Right breast mammogram post-treatment. Fine-linear and branching, calcifications in the right upper-outer breast seen on A) mediolateral-oblique and B) craniocaudal views. Axillary lymph nodes have decreased in size though calcifications remain (arrow). Skin thickening is more easily seen with the digital mammography technique of Figure 3 compared with analog technique used in Figure I. 

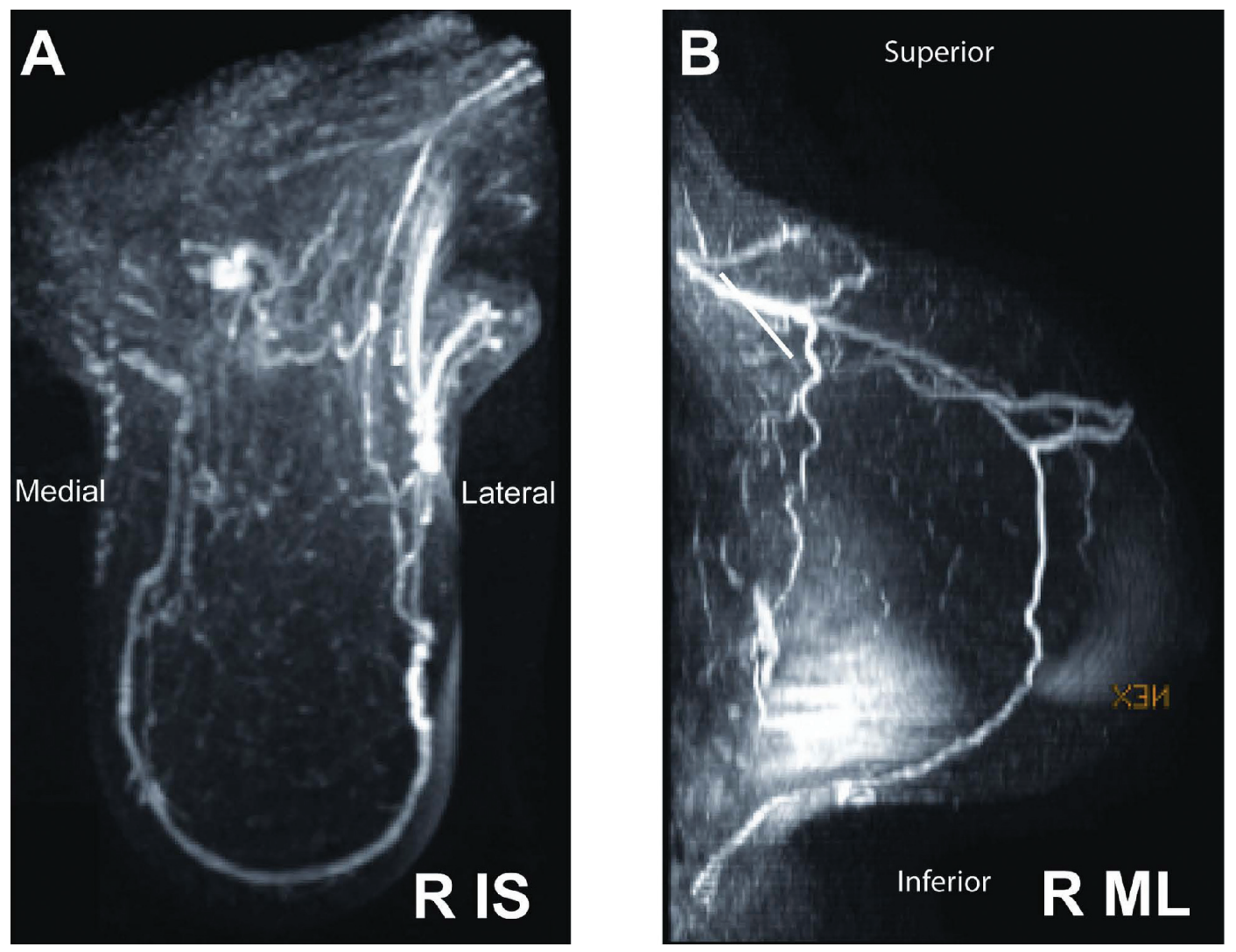

Figure 4 Right breast magnetic resonance imaging post-treatment. Three-dimensional maximal intensity projection reformat of the right breast images in $\mathbf{A}$ ) inferior-superior and B) mediolateral projections. No residual abnormal enhancement was seen in the breast and axillary lymphadenopathy had resolved.

longest diameter are correlated with residual disease at the time of surgery, but there is no correlation between mammography findings and residual pathologic disease. This suggests that calcifications are indicators of disease prior to treatment, but may persist after treatment even when the cancer is eliminated.

The assessment of axillary status is also a key determinant of prognosis and the type of postoperative treatment a patient will require. Preoperative therapy has been shown to clear lymph node disease in $20 \%-40 \%$ of patients. ${ }^{10}$ Furthermore, the National Surgical Adjuvant Breast and Bowel Project (NSABP) B-18 and B-27 studies showed that NAC significantly increased the chance of having pathologically negative lymph nodes. ${ }^{1}$ The presence of axillary disease mandates the performance of a full axillary lymph node dissection. If four or more lymph nodes have tumor invasion, then the patient should also receive radiation therapy. ${ }^{11}$ Unfortunately, both treatments can lead to significant morbidities such as loss of arm mobility, lymphedema, and skin changes. In one study, patients who underwent axillary lymph node dissection had a three-fold increase in pain, a five-fold higher risk of lymphedema, and a three-fold higher risk of impaired use of the arm as compared to patients who underwent sentinel lymph node biopsy (SNB). ${ }^{12}$

Even though SNB is less morbid and equally diagnostic as traditional axillary dissection in adjuvant therapy patients, the accuracy and efficacy of SNB in NAC patients remains controversial. ${ }^{13}$ Potential confounders include lymphatic scarring that affects the usual lymph drainage pattern and selective eradication of disease in the sentinel lymph node, but not in other axillary lymph nodes. Furthermore, whether SNB should be done before or after neoadjuvant therapy is another topic of debate. Results from the I-SPY clinical trial, NSABP B-27, and other studies suggest that post-therapy SNB effectively surveys axillary disease status. ${ }^{14-16}$ However, when there is established disease in axillary lymph nodes pretherapy, SNB may not be sufficient. ${ }^{14}$ More data is required before definitive recommendations may be made.

In our patient, interval MRI showed no remaining disease despite significant residual calcifications. The amount of calcification was difficult to ignore, and its pleomorphic and segmental nature was also highly concerning. Because the patient had matted axillary lymph nodes, we performed a full axillary dissection that surprisingly 
showed no disease in all lymph nodes removed in spite of extensive calcifications. This is a significant finding in that the complete pathologic response in the lymph nodes was accurately assessed by the interval MRI. As imaging technology improves, it would be useful to study prospectively the accuracy of MRI in evaluating axillary lymph nodes in patients receiving NAC.

Even though pleomorphic calcifications are diagnostic for cancer prior to NAC, the persistence of deposits after treatment may be irrelevant and potentially misleading. In our previous studies, we did not assess the performance of MR with respect to the extent of calcifications. ${ }^{3,4,8}$ However, this case is an excellent example demonstrating that calcifications may not resolve after tumor response and the presence of calcifications after NAC should not de facto exclude breast-conserving resections. The case also suggests that MRI may be better than mammography for assessing disease response after neoadjuvant therapy in a tumor with associated calcifications. Similarly, MRI rather than mammography should be considered to monitor for recurrences after breast conservation surgery in patients who have calcifications after NAC. These conclusions need to be confirmed in a larger cohort of patients.

\section{Disclosure}

The authors report no conflicts of interest in this work.

\section{References}

1. Rastogi P, Anderson SJ, Bear HD, et al. Preoperative chemotherapy: updates of National Surgical Adjuvant Breast and Bowel Project Protocols B-18 and B-27. J Clin Oncol. 2008;26:778-785.

2. van Nes JG, Putter H, Julien JP, et al. Preoperative chemotherapy is safe in early breast cancer, even after 10 years of follow-up; clinical and translational results from the EORTC trial 10902. Breast Cancer Res Treat. 2008 May 18. [Epub ahead of print].

3. Esserman L, Hylton N, Yassa L, Barclay J, Frankel S, Sickles E. Utility of magnetic resonance imaging in the management of breast cancer: evidence for improved preoperative staging. J Clin Oncol. 1999;17:110-119.
4. Partridge SC, Gibbs JE, Lu Y, Esserman LJ, Sudilovsky D, Hylton NM. Accuracy of MR imaging for revealing residual breast cancer in patients who have undergone neoadjuvant chemotherapy. AJR Am J Roentgenol. 2002;179:1193-1199.

5. Peintinger F, Buzdar AU, Kuerer HM, et al. Hormone receptor status and pathologic response of HER2-positive breast cancer treated with neoadjuvant chemotherapy and trastuzumab. Ann Oncol. 2008;19(12):2020-2025.

6. Buzdar AU, Ibrahim NK, Francis D, et al. Significantly higher pathologic complete remission rate after neoadjuvant therapy with trastuzumab, paclitaxel, and epirubicin chemotherapy: results of a randomized trial in human epidermal growth factor receptor 2-positive operable breast cancer. J Clin Oncol. 2005;23:3676-3685.

7. Chagpar AB, Middleton LP, Sahin AA, et al. Accuracy of physical examination, ultrasonography, and mammography in predicting residual pathologic tumor size in patients treated with neoadjuvant chemotherapy. Ann Surg. 2006;243:257-264.

8. Partridge SC, Gibbs JE, Lu Y, et al. MRI measurements of breast tumor volume predict response to neoadjuvant chemotherapy and recurrencefree survival. AJR Am J Roentgenol. 2005;184:1774-1781.

9. Hylton NM, Blume JD, Bernreuter WK, et al. Comparison of MRI endpoints for assessing breast cancer response to neoadjuvant treatment: preliminary findings of the American College of Radiology Imaging Network (ACRIN) trial 6657. San Antonio, TX: San Antonio Breast Cancer Symposium; 2008.

10. Kuerer HM, Newman LA, Smith TL, et al. Clinical course of breast cancer patients with complete pathologic primary tumor and axillary lymph node response to doxorubicin-based neoadjuvant chemotherapy. J Clin Oncol. 1999;17:460-469.

11. Recht A, Edge SB, Solin LJ, et al. Postmastectomy radiotherapy: clinical practice guidelines of the American Society of Clinical Oncology. J Clin Oncol. 2001;19:1539-1569.

12. Schijven MP, Vingerhoets AJ, Rutten HJ, et al. Comparison of morbidity between axillary lymph node dissection and sentinel node biopsy. Eur J Surg Oncol. 2003;29:341-350.

13. Buchholz TA, Lehman CD, Harris JR, et al. Statement of the science concerning locoregional treatments after preoperative chemotherapy for breast cancer: a National Cancer Institute conference. J Clin Oncol. 2008;26:791-797.

14. Gomez RE, Zakhireh J, Moore D, et al. Sentinel node biopsy performed in the neoadjuvant setting for breast cancer: results from the I-SPY TRIAL (CALGB 150007/150012 and ACRIN 6657). San Antonio, TX: San Antonio Breast Cancer Symposium; 2008.

15. Mamounas EP, Brown A, Anderson S, et al. Sentinel node biopsy after neoadjuvant chemotherapy in breast cancer: results from National Surgical Adjuvant Breast and Bowel Project Protocol B-27. J Clin Oncol. 2005;23:2694-2702.

16. Lang JE, Esserman LJ, Ewing CA, et al. Accuracy of selective sentinel lymphadenectomy after neoadjuvant chemotherapy: effect of clinical node status at presentation. J Am Coll Surg. 2004;199:856-862. 